03

\title{
Внутренние волны плотности ударного типа, индуцированные хемоконвекцией в смешивающихся реагирующих жидкостях
}

\author{
(ㄱ Д.А. Брацун
}

Пермский национальный исследовательский политехнический университет

E-mail: DABracun@pstu.ru

Поступило в Редакцию 21 июня 2017 г.

Дается теоретическое объяснение явления спонтанного возникновения волн плотности, обнаруженных недавно экспериментально в двухслойных системах смешивающихся жидкостей, помещенных в узкий вертикальный зазор ячейки Хеле-Шоу в поле тяжести. Верхний и нижний слои представляют собой соответственно водные растворы кислоты и основания, при контакте которых начинает протекать реакция нейтрализации. Процесс сопровождается сильной зависимостью коэффициентов диффузии реагентов от их концентрации, что приводит к возникновению локальных карманов плотности, в которых развивается конвекция. При определенных условиях полости схлопываются, порождая скачок плотности, который перемещается быстрее характерных возмущений в среде и имеет вид ударной волны. Предложена математическая модель явления, которая при определенных допущениях может быть формально сведена к уравнениям движения сжимаемого газа. Представлены численные расчеты, проводится сравнение с данными экспериментов.

DOI: 10.21883/PJTF.2017.20.45152.16927

В последние годы внимание большого числа исследователей приковано к изучению взаимного влияния конвективных течений и химических реакций. Этот интерес стимулируется важными технологическими приложениями, среди которых выделим нефтепереработку [1], фотополимеризацию [2], внешнее управление [3], хемосорбцию [4]. Выяснилось, что реакции могут приводить к существенным изменениям плотности, вязкости, диффузии растворов. В свою очередь эти изменения способствуют формированию структур нового типа, 
резко меняющих распределение реагентов. Среди реакций выделяется реакция нейтрализации кислоты основанием, которая обладает сравнительно простой, хотя и нелинейной, кинетикой. Для смешивающихся растворов главной причиной развития хемоконвекции выступают гравитационные механизмы движения, обусловленные зонами неустойчивой стратификации плотности вследствие разной скорости диффузии реагентов [5]. В работе [6] была сделана попытка общей классификации всех возможных видов неустойчивости, основанная на изучении асимптотики больших времен эволюции системы. Были выделены неустойчивости двойной диффузии (DD-конвекция), диффузионного слоя (DLC-конвекция) и Рэлея-Тейлора (RT-конвекция). Однако в работах автора с коллегами [7,8] было показано, что такая классификация не является полной, так как, вообще говоря, коэффициенты диффузии реагентов зависят от концентрации растворов, что может приводить к полной перестройке профиля плотности в ходе реакции. Это может приводить к возникновению неустойчивости, локализованной в карманах плотности, которую авторы назвали конвекцией концентрационно-зависимой диффузии (CDD-конвекция) [7]. Она представляет собой периодическую систему хемоконвективных ячеек, формирующихся параллельно фронту реакции и перпендикулярно направлению силы тяжести [8].

Настоящая работа посвящена теоретическому объяснению явления, обнаруженного недавно экспериментально в той же системе смешивающихся реагирующих растворов. В [9] было показано, что при определенном сочетании начальных концентраций реагентов, близких к изопикне, CDD-конвекция может внезапно смениться волной плотности, которая имеет плоский фронт и распространяется в направлении вектора силы тяжести со скоростью, на порядок превышающей скорость диффузионных процессов. В данной работе дается теоретическое объяснение этого явления.

Пусть два слоя смешивающихся жидкостей помещены в вертикальную ячейку Хеле-Шоу, зазор между широкими гранями которой равен $h$. Хотя эффект обнаружен для различных пар веществ [9], для определенности будем считать, что верхний слой представляет собой водный раствор азотной кислоты $\mathrm{HNO}_{3}$ с начальной концентрацией $A_{0}$, а нижний - водный раствор гидроксида натрия $\mathrm{NaOH}$ с концентрацией $B_{0}$. При приведении растворов в контакт начинается реакция нейтрализации, которая характеризуется скоростью $k$ и приводит к

Письма в ЖТФ, 2017, том 43, вып. 20 
возникновению соли $C$. Отметим, что описанный процесс является неавтономным, так как в ходе реакции реагенты не пополняются, а профили концентраций необратимо меняются. Пусть координаты $x$ и $z$ проведены вдоль широких граней ячейки Хеле-Шоу таким образом, что линия $z=0$ определяет начальную контактную поверхность между слоями, а сила тяжести и ось $z$ направлены в разные стороны. Выбирая в качестве единиц измерения длины $h$, времени $h^{2} / D_{a 0}$, скорости $D_{a 0} / h$ и концентрации $A_{0}$, где $D_{a 0}-$ табличное значение коэффициента диффузии азотной кислоты в воде при температуре $25^{\circ} \mathrm{C}, \rho_{0}$ и $v-$ плотность и коэффициент кинематической вязкости воды, получим систему уравнений конвекции-реакции-диффузии в приближении Хеле-Шоу

$$
\begin{gathered}
\frac{1}{S c}\left(\frac{\partial \Phi}{\partial t}+\frac{6}{5} \frac{\partial(\Psi, \Phi)}{\partial(z, x)}\right)=\nabla^{2} \Phi-12 \Phi-\frac{\partial \rho}{\partial x} \\
\frac{\partial A}{\partial t}+\frac{\partial(\Psi, A)}{\partial(z, x)}=\nabla D_{a}(A) \nabla A-\alpha A B, \\
\frac{\partial B}{\partial t}+\frac{\partial(\Psi, B)}{\partial(z, x)}=\nabla D_{b}(B) \nabla B-\alpha A B, \\
\frac{\partial C}{\partial t}+\frac{\partial(\Psi, C)}{\partial(z, x)}=\nabla D_{c}(C) \nabla C-\alpha A B, \\
\rho=R_{a} A+R_{b} B+R_{c} C .
\end{gathered}
$$

Здесь использована двухполевая запись уравнения движения (1) и введены в рассмотрение функция тока $\Psi$ и завихренность $\Phi=-\Delta \Psi$, а выражения (2)-(4) записаны с учетом кинетики реакции нейтрализации. Ранее было обнаружено [7], что зависимость коэффициентов диффузии реагентов от концентраций критически влияет на эволюцию системы. Для пары $\mathrm{HNO}_{3} / \mathrm{NaOH}$ на основе эмпирических данных были построены линейные аппроксимации зависимостей, дающие приемлемый результат вплоть до концентраций $3 \mathrm{~mol} / \mathrm{l}$ :

$$
\begin{gathered}
D_{a}(A) \approx 0.158 A+0.881, \quad D_{b}(B) \approx-0.087 B+0.594, \\
D_{c}(C) \approx-0.284 C+0.478 .
\end{gathered}
$$

К уравнениям (1)-(6) следует добавить стандартные граничные условия (прилипание для скорости, отсутствие потока для реагентов), а

Письма в ЖТФ, 2017, том 43, вып. 20 
также начальные условия

$$
\begin{gathered}
z<0: \quad \Psi=0, \Phi=0, A=0, B=B_{0} / A_{0}, C=0, \\
z \geq 0: \quad \Psi=0, \Phi=0, A=1, B=0, C=0 .
\end{gathered}
$$

В задаче (1)-(7) появились безразмерные параметры: число Шмидта $S c=v / D_{a 0}$, число Дамкёлера $\alpha=k A_{0} h^{2} / D_{a 0}$, концентрационные числа Рэлея $R_{i}=g \beta_{i} A_{0} h^{3} / v D_{a 0} \quad(i=a, b, c)$. Для $\mathrm{HNO}_{3} / \mathrm{NaOH}$ эти параметры можно оценить как $S c=317, R_{a}=3.2 \cdot 10^{5}, R_{b}=3.8 \cdot 10^{5}$, $R_{c}=5.1 \cdot 10^{5}$. Поскольку реакция нейтрализации протекает быстро, положим $\alpha \approx 10^{3}$. Таким образом, единственным свободным параметром задачи остается $B_{0} / A_{0}$. Задача $(1)-(7)$ решалась численно методом конечных разностей, детали которого изложены в $[7,8]$.

Приведем некоторые результаты расчетов. Геометрия области соответствует параметрам кюветы из работы [9]. На рис. 1 представлена карта устойчивости хемоконвективных движений на плоскости начальных значений концентраций кислоты $A_{0}$ и основания $B_{0}$, обезразмеренных с помощью соответствующих коэффициентов объемного расширения $\beta_{a}$ и $\beta_{b}$. Поскольку в данной работе рассматриваются только изначально устойчивые системы, интерес представляет часть карты, которая находится выше изопикны. В этом случае верхний слой легче, и опрокидывания системы не происходит. Конвенциональная теория [6] предсказывает для данной двухслойной системы растворов $\mathrm{HNO}_{3}$ и $\mathrm{NaOH}$ (кислота вверху) возникновение DLC-неустойчивости. Она представляет собой формирование двух областей слабых конвективных движений, разделенных диффузионной зоной, в которой жидкость остается неподвижной. Выше и ниже зоны возникают системы солевых пальцев, которые распространяются соответственно вверх и вниз от фронта реакции [6]. Карта устойчивости на рис. 1 показывает, что кроме DLC из-за эффекта зависимости коэффициентов диффузии реагентов от концентрации вблизи изопикны могут возникать еще два неизвестных ранее режима конвекции. Неустойчивость CDD возникает, когда на профиле плотности появляется локальный минимум (рис. 1, точка 2 и профиль плотности 2). Причина его появления обсуждалась в работах [7,8]: скорость диффузии соли (6) быстро уменьшается с ростом ее концентрации, что приводит к локальному накоплению продукта реакции в зоне реакции. Так как соль достаточно тяжелая, ее вклад в плотность (5) существен, что 

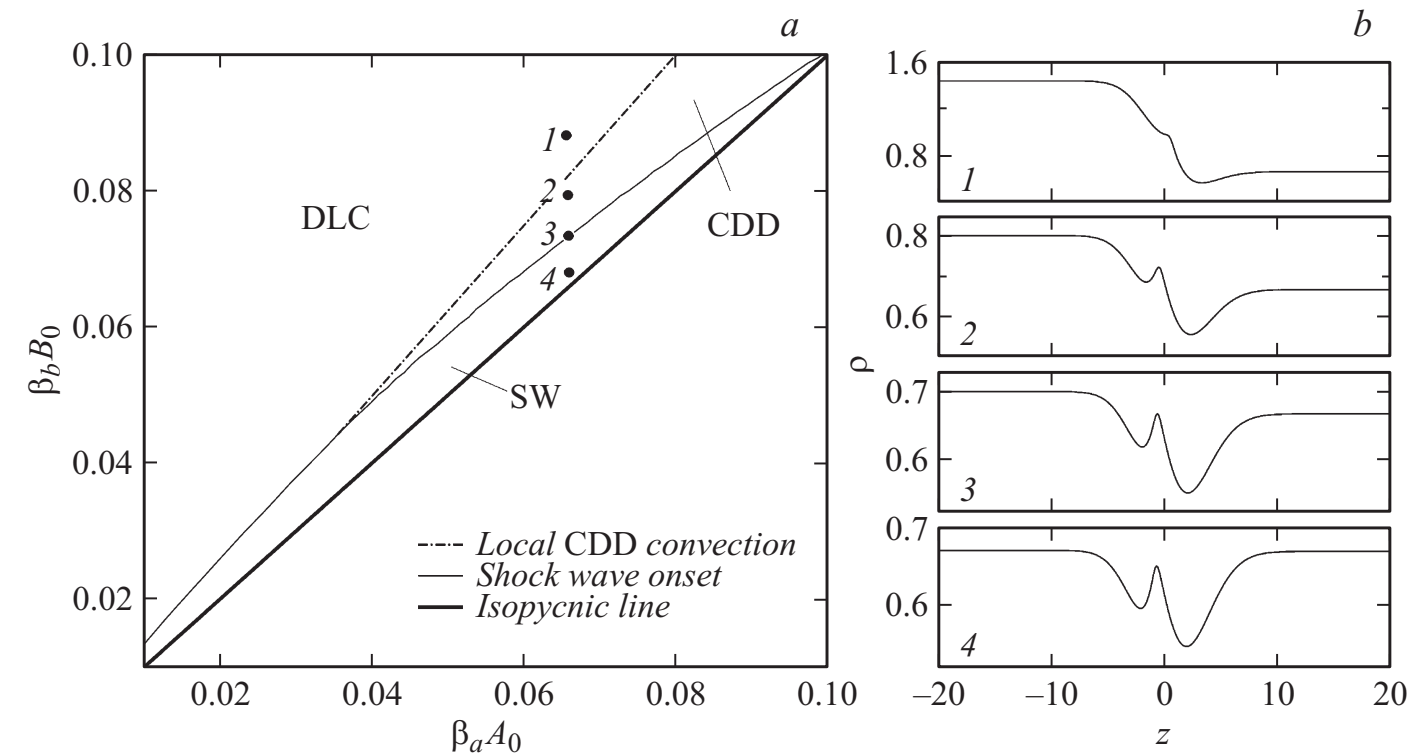

Рис. 1. $a-$ карта режимов хемоконвективных движений на плоскости параметров $\beta_{a} A_{0}$ и $\beta_{b} B_{0}$. Отмечены области конвекции диффузионного слоя (DLC), неустойчивости концентрационно-зависимой диффузии (CDD) и возникновения волны плотности ударного типа $(\mathrm{SW}) . b-$ характерный вид профиля плотности $\rho$ в случае отсутствия конвекции для разных значений параметров, отмеченных на карте устойчивости. 


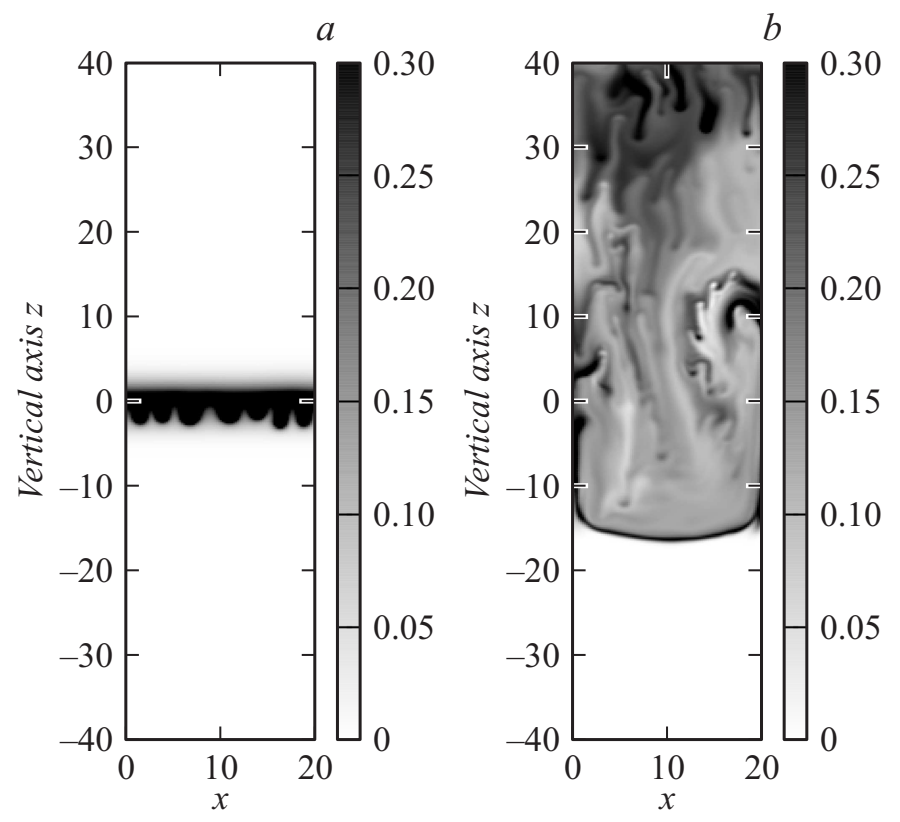

Рис. 2. Мгновенное поле концентрации соли $C$ для режима CDD конвекции $(a)$ и распространения ударной волны плотности $(b)$ в момент времени $t=2$. Поля представляют собой результат нелинейного развития конвективных возмущений для значений параметров, отмеченных на карте устойчивости цифрами 2 и 4 соответственно.

приводит к возникновению локального максимума плотности. Из-за неустойчивой стратификации плотности внутри плотностных карманов возникает хемоконвекция, которая представляет собой систему периодических ячеек, зажатых между двумя горизонтальными слоями неподвижной жидкости (рис. 2,a). Такая циркуляция может продолжаться квазистационарно достаточно долго (в эксперименте [9] процесс наблюдался часами) до полного расходования исходных реагентов.

При задании начальных концентраций реагентов еще ближе к изопикне возникает удивительная структура, которая ранее не наблюдалась. Из рис. 1 (точка 3 и профиль плотности 3) видно, что новый 
режим появляется тогда, когда плотности реакционной зоны и верхнего слоя становятся равными. В этом случае при малейшем изменении параметров в сторону изопикны в системе стремительно развивается волна плотности, профиль которой меняется скачкообразно, а сама она быстро распространяется в направлении силы тяжести (на рис. 2, $b$ представлен лишь один кадр эволюции). Волна возникает в результате схлопывания карманов плотности с CDD-конвекцией внутри. Скорость распространения волны в расчетах составляет примерно $0.06 \mathrm{~mm} / \mathrm{s}$ $\left(A_{0}=2 \mathrm{~mol} / 1, B_{0}=1.7 \mathrm{~mol} / \mathrm{l}\right)$, что хорошо согласуется с экспериментальными данными [9]. Отметим, что фронт волны во время движения остается плоским, что резко контрастирует с привычной для хемоконвективных задач фингер-неустойчивостью. При этом волна демонстрирует мощное перемешивание всех ингредиентов в своем спутном потоке (рис. 2, $b$ ). Весь процесс прохождения волны по кювете и полная реакция исходных реагентов занимает в эксперименте всего 5-7 min. Также не следует путать наблюдаемое явление с волнами детонации, которые распространяются со сверхзвуковыми скоростями. Можно показать, однако, что уравнения (1)-(5) при определенных допущениях могут быть формально сведены к уравнениям газодинамики, в которых решения в виде ударных волн могут быть получены аналитически. Можно заметить (рис. 2,b), что процессы диффузии в ударной волне играют пренебрежимо малую роль, так как основным двигателем для нее является система мощных вихрей в спутном потоке. Таким образом, процессы диффузии в уравнениях можно не учитывать. Кроме того, удобно рассматривать вместо реакции нейтрализации реакцию первого порядка $A \rightarrow C$, которая является ее грубой моделью. Отказываясь для простоты от приближения Хеле-Шоу, умножим уравнения (2), (4) на соответствующие числа Рэлея, взятые на изопикне, и сложим их. После несложных преобразований получим

$$
\begin{gathered}
\frac{\partial \Phi}{\partial t}+v_{x} \frac{\partial \Phi}{\partial x}+v_{z} \frac{\partial \Phi}{\partial z}+S c \frac{\partial \rho}{\partial x}=0 \\
\frac{\partial \rho}{\partial t}+\frac{\partial \rho v_{x}}{\partial x}+\frac{\partial \rho v_{z}}{\partial z}=0
\end{gathered}
$$

где $v_{x}, v_{z}$ - компоненты скорости жидкости. Уравнения (8) формально совпадают с уравнениями гравитационных поверхностных волн в при-

Письма в ЖТФ, 2017, том 43, вып. 20 
ближении „мелкой воды““ $[10]$

$$
\frac{\partial v}{\partial t}+v \frac{\partial v}{\partial x}+g \frac{\partial h}{\partial x}=0, \quad \frac{\partial h}{\partial t}+\frac{\partial v h}{\partial x}=0
$$

где $v$ - скорость жидкости, $h$ - высота уровня воды. Таким образом, роль скорости в (8) выполняет завихренность, а роль высоты плотность. Как известно, задача о поверхностных волнах в приближении „мелкой воды“ математически является полной аналогией адиабатического движения идеального газа с показателем адиабаты $\gamma=2$ [10]

$$
\frac{\partial v}{\partial t}+v \frac{\partial v}{\partial x}+c^{2} \frac{\partial \rho}{\partial x}=0, \quad \frac{\partial \rho}{\partial t}+\frac{\partial v \rho}{\partial x}=0,
$$

где $c-$ скорость звука. Понятно, что скорость распространения ударной волны в (9) не сверхзвуковая, как в газодинамике (10), а определяется комплексом $\sqrt{g h}$. Таким образом, рассматриваемая в нашей работе система (8) является еще одной математической аналогией для ударных волн в газодинамике. Сравнивая (8), (9) и (10), заключаем, что роль критической скорости в нашей задаче выполняет комплекс

$$
V_{\text {shock }}=\sqrt{S c} \approx 17.8,
$$

что в размерных единицах составляет примерно $0.056 \mathrm{~mm} / \mathrm{s}$. Это значение находится в превосходном согласии с данными экспериментов и нашими расчетами. Более того, расчеты и эксперименты показывают, что, если волна начинает двигаться медленнее, чем (11), она останавливается, запуская диффузионные механизмы неустойчивости. Отметим, что наша система является более близкой аналогией, так как волны в (8) и (10) продольные, а волны на поверхности воды (9) поперечные.

Работа поддержана грантом Министерства образования и науки РФ (№ 3.6990.2017/БЧ).

\section{Список литературы}

[1] Wei C., Ortoleva P. // Earth Sci. Rev. 1990. N 29. P. 183-198.

[2] Belk M., Kostarev K., Volpert V., Yudina T. // J. Phys. Chem. B. 2003. V. 107. P. 10292-10298.

[3] Брачун Д.А., Де Вит А. // ЖТФ. 2008. Т. 78. В. 2. С. 6-13.

Письма в ЖТФ, 2017, том 43, вып. 20 
[4] Карлов С.П., Казенин Д.А., Баранов Д.А., Волков А.В., Полянин Д.А., Вязьмин А.В. // ЖФХ. 2007. Т. 81. № 5. С. 775-791.

[5] Almarcha C., Trevelyan P.M.J., Grosfils P., De Wit A. // Phys. Rev. Lett. 2010. V. 104. N 4. P. 044501.

[6] Trevelyan P.M.J., Almarcha C., De Wit A. // Phys. Rev. E. 2015. V. 91. P. 023001.

[7] Bratsun D., Kostarev K., Mizev A., Mosheva E. // Phys. Rev. E. 2015. V. 92. P. 011003.

[8] Bratsun D.A., Stepkina O.S., Kostarev K.G., Mizev A.I., Mosheva E.A. // Microgravity Sci. Technol. 2016. V. 28. N 3. P. 575-585.

[9] Мизев А.И., Мошева Е.А., Костарев К.Г., Лысенко С.Н. // Конвективные течения / Под ред. В.Г. Козлова. Пермь: ПГПУ, 2015. В. 7. С. 158-175.

[10] Ландау Л.Д., Лифиии, Е.М. Гидродинамика. М.: Наука, 1986. 736 с. 